OPEN

SUBJECT AREAS:

CHARACTERIZATION AND ANALYTICAL

TECHNIQUES

OPTICAL SENSORS

IMAGING AND SENSING

Received

6 June 2014

Accepted

15 August 2014

Published

15 September 2014

Correspondence and requests for materials should be addressed to

K.D.K. (kkihm@utk.

edu)

\section{How to Reliably Determine the Complex Refractive Index (RI) of Graphene by Using Two Independent Measurement Constraints}

\author{
Sosan Cheon', Kenneth David Kihm, ${ }^{1,}$, Hong goo Kim³, Gyumin Lim', Jae Sung Park³ \& Joon Sik Lee ${ }^{1,3}$
}

\begin{abstract}
'Multiscale Mechanical Design Division, World Class University Program, Seoul National University, Seoul 151-744, South Korea,
${ }^{2}$ Mechanical, Aerospace, and Biomedical Engineering, the University of Tennessee, Knoxville, Tennessee 37996, USA,

${ }^{3}$ Department of Mechanical and Aerospace Engineering, Seoul National University, Seoul 151-744, South Korea.
\end{abstract}

Reliable determination of the complex refractive index (RI) of graphene inherently requires two independent measurement realizations for two independent unknowns of the real $\left(n_{G}\right)$ and imaginary $\left(k_{G}\right)$ components, i.e., $\mathrm{RI}=n_{G}+i k_{G}$. Thus, any single set of measurement realization provides only one constraint that is insufficient to uniquely determine the complex RI of graphene. Tandem uses of two independent measurement techniques, namely the surface plasmon resonance (SPR) angle detection and the attenuated total reflection (ATR) intensity measurement, allow for the unique determination of the complex RI of CVD-synthesized graphene. The presently measured graphene RI is determined to be $2.65+1.27 \mathrm{i}$ for the E-field oscillating parallel to graphene at $634 \mathrm{~nm}$ wavelength, with variations for different numbers of $L(1,3$ and 5) remaining within $\pm 3 \%$. Thus, our demonstration results for the specified wavelength serve as an impetus to suggest the need for two independent measurement techniques in determining both the real and imaginary RI values for graphene. Additional efforts have been made to characterize graphene layers using the density function theory (DFT): this calculation provides $\mathrm{RI}_{G}=2.71+1.41$.

T he relatively significant light absorption of $2 \sim 3 \%$ per single graphene layer ${ }^{1}$ of mere $0.335 \mathrm{~nm}$ implies that the refractive index (RI) of graphene must be complex with a significant imaginary component. Notable efforts (Table 1) have been made to determine the complex graphene RI using diverse experimental and theoretical approaches ${ }^{2-12}$, yet the available data show substantial scattering for both their real and imaginary components. Some of this scattering may be attributed to the graphene sample variations and/or different sample preparation processes, as well as to the different measurement uncertainties associated with the different measurement techniques. However, the more fundamental origins of the large data scattering are considered to be two-fold: (1) the graphene RI is complex-valued, having two unknowns of both real and imaginary parts at a given wavelength, and (2) the ultra-thin graphene layers can be naturally better characterized by using a probe focusing on the near-field. To uniquely determine both the real and imaginary parts of graphene RI, therefore, two independent sets of measurement observables will be necessary, using two discrete measurement techniques for the same graphene sample. Furthermore, probing near-field characterization will enhance the measurement sensitivity, since the surface-enhanced electromagnetic field exists enclosing the thin graphene layer.

Employing two independent techniques requires two separate experimental systems as well as two different sample layouts and preparations. In contrast, notable efforts have been made by fitting experimental data from a single measurement technique to a dispersion model for RI of graphene. The earlier use of reflection spectroscopy ${ }^{2}$ imposed an overly simplified assumption of both real and imaginary parts of the complex RI being constant over the visible range, whereas its later use ${ }^{3}$ modeled the imaginary part of the complex RI using a constant optical conductivity based on the earlier report ${ }^{1}$, yet retained an assumption of the real part of RI as a constant.

Ellipsometry measures two variables (the phase and the intensity of reflected waves); however, direct inversion of these two measured variables into real and imaginary RI components usually requires a complex fitting process since all of the four variables are implicitly interconnected in Fresnel's multilayer reflectance equation. Spectroscopic ellipsometry ${ }^{4-6}$ uses a more elaborate dispersion modeling for the spectroscopic data inversion. 


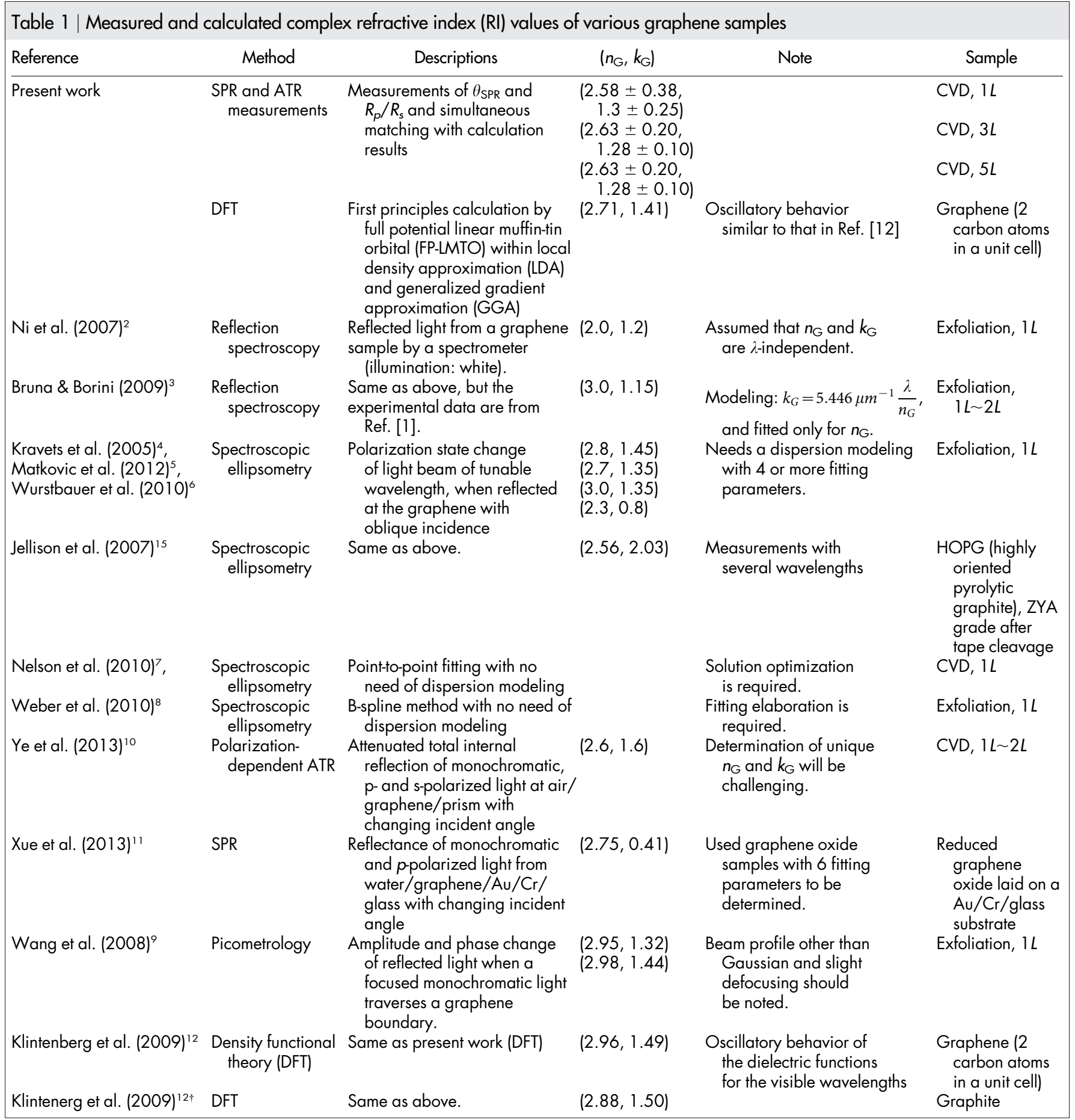

However, the reliability of the optimized result can depend on the stiffness of each variable and the orthogonalities between variables in fitting. More recent work on ellipsomery ${ }^{7,8}$ successfully demonstrated that assumptions on the dispersion relation modeling can be released, either by very precise measurements to obtain smooth spectral curves for the "point-by-point fitting" result, or by novel Bspline fitting, which doesn't require any physical assumption in spectroscopic data inversion process.

As a non-spectroscopic method, which does not require a dispersion modeling, picometrology ${ }^{9}$ uses both the amplitude and phase change as two observables when light traverses the graphene edge area, but the scheme bears several error sources creating uncertainties that are not quantitatively resolved. As another non- spectroscopic method, the polarization dependence of optical absorptions under the internal reflection condition was measured to determine the complex RI by fitting ${ }^{10}$, however, the uniqueness of the fitted real and imaginary parts of RI is somewhat questionable because of the single constraint of the polarization dependence. For the case of a graphene flake oxide sample, fitting of the surface plasmon reflectance curves ${ }^{11}$ was attempted to determine the complex RI, but again further validation will be needed for the uniqueness of the fitted results because of too many fitting parameters (6) for the single observable.

In this report, we demonstrate successful implementation of a reliable and robust way to determine the complex RI of graphene using two independent and non-spectroscopic measurement tech- 
$\mathbf{a}$

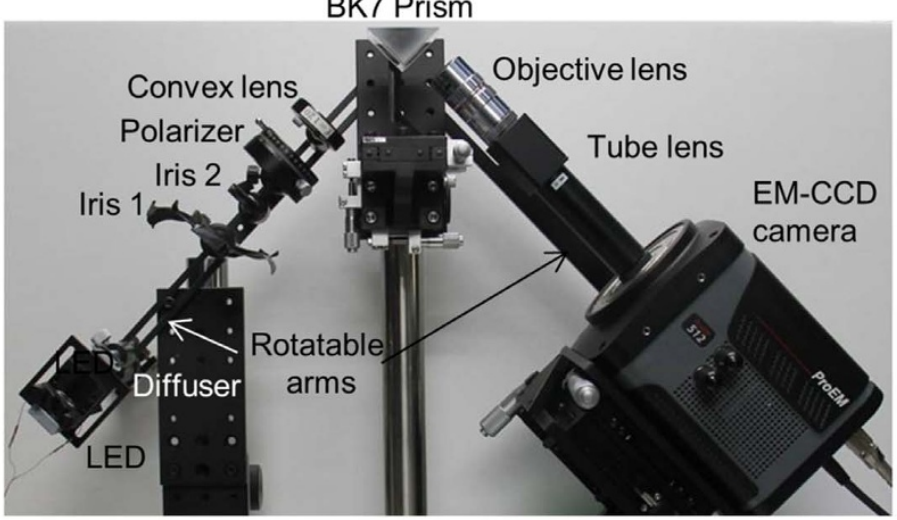

b

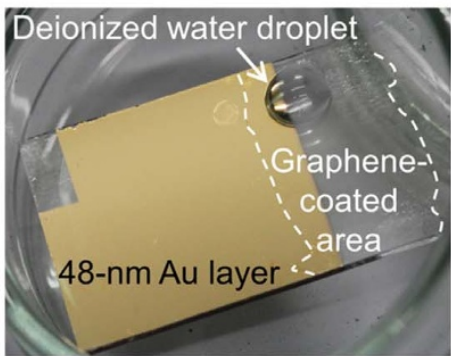

d

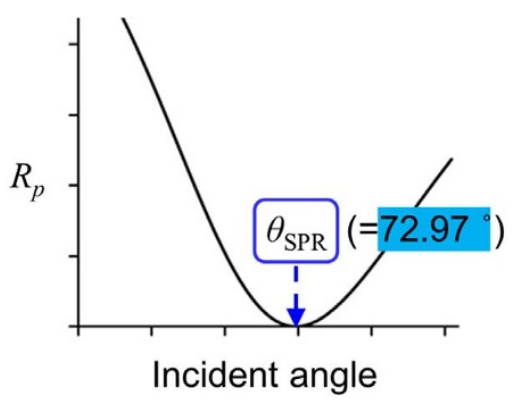

f

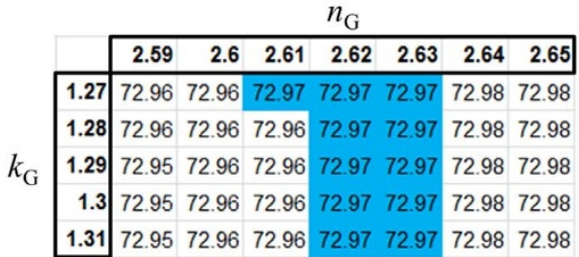

c

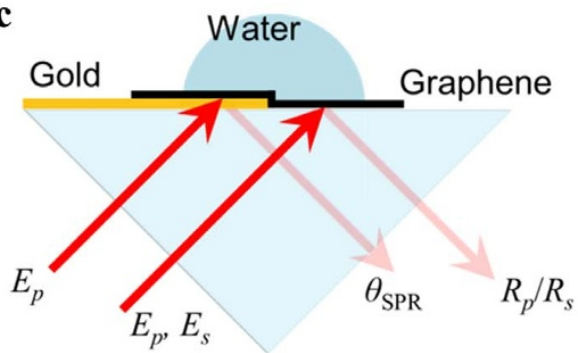

e

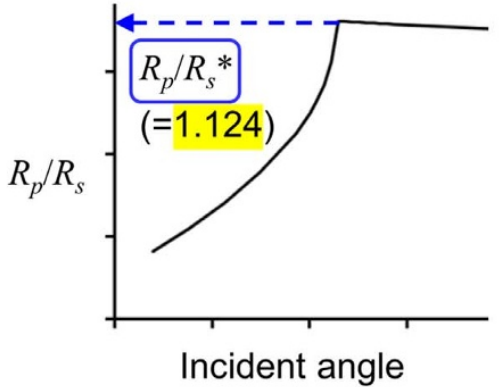

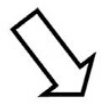

g
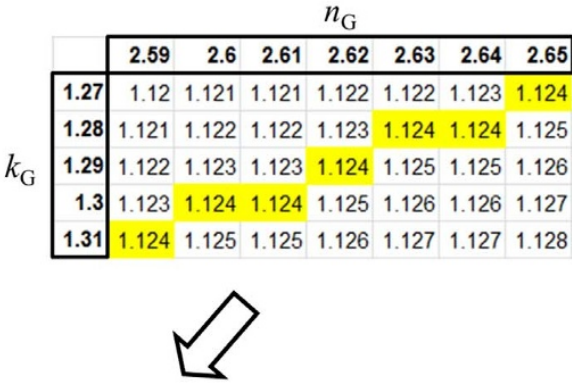

h

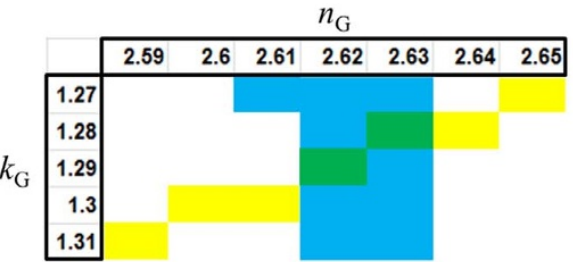

Figure 1 How to reliably measure the refractive index (RI) of graphene. (a) The experimental layout to determine the complex RI of graphene layers using two independent measurement realizations for the SPR angle $\left(\theta_{\mathrm{SPR}}\right)$ and the maximum reflectance ratio $R_{p} / R_{s}$, (b) a graphene sample laid on a BK7 glass substrate that is partially coated with 48 -nm thick Au film, (c) schematic of the two tandem measurements for $\theta_{\mathrm{SPR}}$ and $R_{p} / R_{s}$ under a deionized water environment (in lieu of air) for enhanced measurement sensitivities, (d) $\theta_{\mathrm{SPR}}=72.97^{\circ}$ is measured from the maximum resonant absorption of surface plasmon polaritons (SPPs), or equivalently as the minimum reflectance of the p-polarized incident ray $(634 \mathrm{~nm})$, (e) the maximum $R_{p} / R_{s}=1.124$ is measured at the critical angle for total internal reflection, (f) all possible fitting solutions of $\left(n_{G}, k_{G}\right)$ that satisfy the first constraint of $\theta_{\mathrm{SPR}}=72.97^{\circ}$, (g) all possible fitting solutions of $\left(n_{G}, k_{G}\right)$ that satisfy the second constraint of maximum $R_{p} / R_{s}=1.124$, and (h) determination of the unique complex RI of graphene, $\mathrm{RI}_{G}=\left(n_{G}, k_{G}\right)=2.63+1.28 i$ from the intersection of $(\mathrm{f})$ and $(\mathrm{g})$, which simultaneously satisfies the two constraints. 


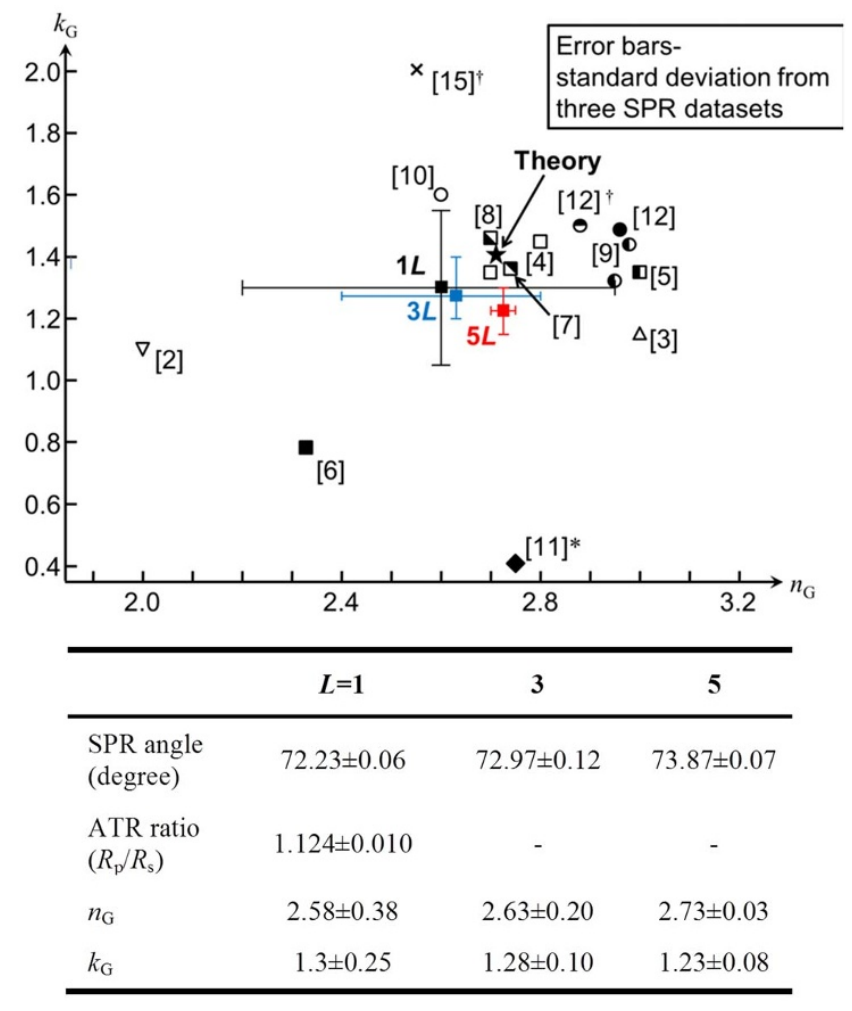

Figure $2 \mid$ Measured and predicted $\mathrm{RI}_{G}$ in comparison with other published results. The three measured $\mathbf{R I}_{\boldsymbol{G}}$ data respectively for $1 L, 3 L$, and $5 L$ graphene samples show deviations of a mere $\pm 3 \%$ from the averaged $\mathbf{R I}_{G}=\mathbf{2 . 6 5}+\mathbf{1 . 2 7 i}$. The extent of the error bars decreases with increasing $L$, primarily because of the enhanced measurement sensitivities of $\theta_{\mathrm{SPR}}$ with thicker graphene samples while the measurement uncertainty for $R_{p} / R_{s}$ remains unchanged. The predicted result of $\mathrm{RI}_{G}=\mathbf{2 . 7 1}+\mathbf{1 . 4 1 i}(\star)$ using the density function theory (DFT) agrees fairly well with all of the measured $\mathrm{RI}_{G}$ data. $\mathrm{RI}_{G}$ data from previous efforts include the reflection spectroscopy ${ }^{2,3}$, the spectroscopic ellipsometry for graphene ${ }^{4-6}$ and for highly oriented pyrolytic graphite ${ }^{15 \dagger}$, more recent ellipsometry that alleviate the need of dispersion modeling ${ }^{7,8}$, the picometrology ${ }^{9}$, absorption under internal reflection ${ }^{10}$, surface plasmon reflectance for reduced graphene oxide $(\mathrm{rGO})^{11 *}$, DFT calculations for graphen $\mathrm{e}^{12}$ and for graphite ${ }^{12 \dagger}$. More detailed descriptions on these published data are shown in Table 1, and further elaboration on their uncertainties is presented in the main text. ( ${ }^{\dagger}$ : Results for graphite samples; *: Results for reduced graphene oxide sample; All other data: Results for CVD-synthesized or exfoliated graphene samples). niques: (1) detection of the maximum absorption of the $p$-polarized incidence at surface plasmon resonance (SPR) angle $\theta_{\mathrm{SPR}}$ by surface plasmon polaritons (SPPs) of a thin Au layer coated with graphene, and (2) detection of the reflectance ratio of $R_{\mathrm{p}} / R_{\mathrm{s}}$ at the critical angle when the incident light is attenuated by the graphene sample without an $\mathrm{Au}$ interlayer. These two independent observables allow for the unique determination of the real and imaginary parts of the complex $\mathrm{RI}$ of graphene. The main point that we would like to report here is that the proposed tandem technique, unlike any previously published techniques, can provide two independently measured variables, allowing the straightforward determination of both the real and imaginary components of graphene RI with no fitting elaboration.

\section{Results}

The main design of the experimental system is comprised of two rotatable arms and a prism assembly placed near the pivot point (Fig. 1a): (1) the left arm consists of the incident LED light source of $634 \mathrm{~nm}$ wavelength with a FWHM (full width half maximum) of $22 \mathrm{~nm}$, a polarizer and collimating optics, (2) the right arm consists of an EMCCD (electron-multiplied charge-coupled device) camera and microscopic lens assembly, and (3) the top surface of the BK7 prism accommodates an index matching BK7 glass slide that is partially coated with a 48 -nm thick gold layer (Fig. 1b). The gold coated surface is used to measure $\theta_{\mathrm{SPR}}$ and the uncoated BK7 glass surface is used to determine the maximum $R_{\mathrm{p}} / R_{\mathrm{s}}$ (Fig. 1c). Note that the water environment is provided for the graphene sample to enhance the angular measurement sensitivity by increasing the magnitude of the SPR angle ranges, in comparison to an air environment ${ }^{13}$.

When the surface plasmon polaritons (SPPs) - the surface charge density waves of the $p$-polarized incident light - are resonantly coupled with the free electrons in the Au layer, the resulting reflectance $R_{p}$ falls to a minimum ${ }^{14}$ at $\theta_{\mathrm{SPR}}$ (Fig. 1d). For the four-layered structure (BK7 glass-48 nm Au layer-graphene layer-water), $\theta_{\mathrm{SPR}}$ is given as a function of both the real $\left(n_{G}\right)$ and imaginary $\left(k_{G}\right)$ components of RI of graphene (Fig. 1f) based on the modified Fresnel equations (see the first Supplementary note online). For the case of $3 L$, for instance, the experimentally measured $\theta_{\mathrm{SPR}}=72.97^{\circ}$ satisfies all combinations of $\left(n_{G}, k_{G}\right)$ as marked in blue in Fig. 1f, and thus, it is clear that the determination of a unique set of $n_{G}$ and $k_{G}$ requires an additional constraint from another independently measured set of data.

The additional constraint comes from the measurements of the maximum $R_{\mathrm{p}} / R_{\mathrm{s}}$ as schematically illustrated in Fig. 1c. When an absorbing layer such as graphene disjoins the water-glass (BK7) interface, which is subjected to total internal reflection, the evan- a

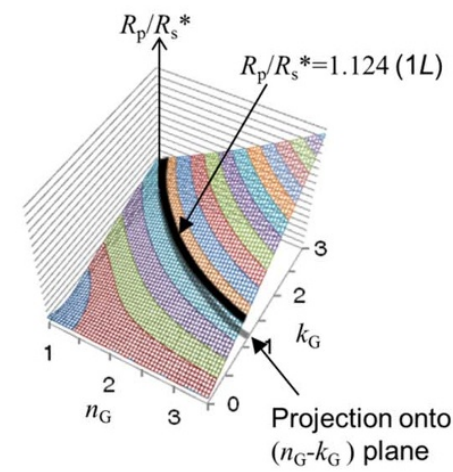

b

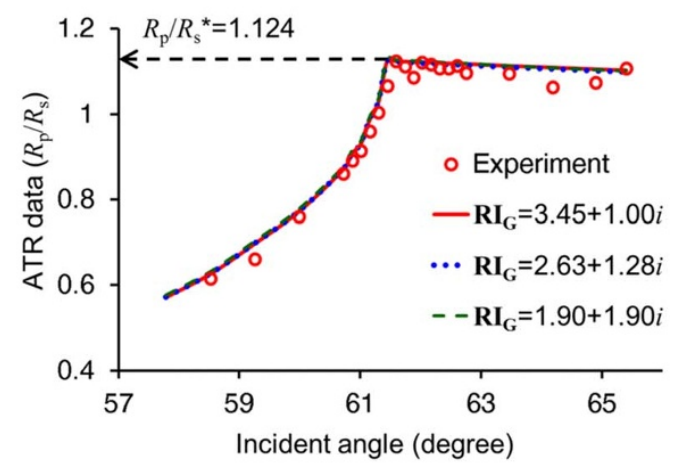

Figure $3 \mid$ Uncertainties associated with the single constraint of $R_{p} / R_{s}$ data. (a) The measured maximum $R_{p} / R_{s}$, i.e., $R_{p} / R_{s}^{*}=1.124$, is shown as the solid curve (black) on the calculated 3-D contour (see second Supplementary note online), and its projection onto the ( $\left.n_{G}, k_{G}\right)$ plane (the gray curve) corresponds to all of the fitted solution candidates for $\mathrm{RI}_{G}=n_{G}+i k_{G}$, under the given single constraint, and (b) three arbitrarily selected pairs of ( $n_{G}$, $k_{G}$ ) among these solution candidates predict an identical $R_{p} / R_{s}$ curve (see second Supplementary note online) and all of them fit to the experimental $R_{p} / R_{s}$ data, which demonstrates the difficulty in uniquely determining the complex RI of graphene using the $R_{p} / R_{s}$ data alone. 


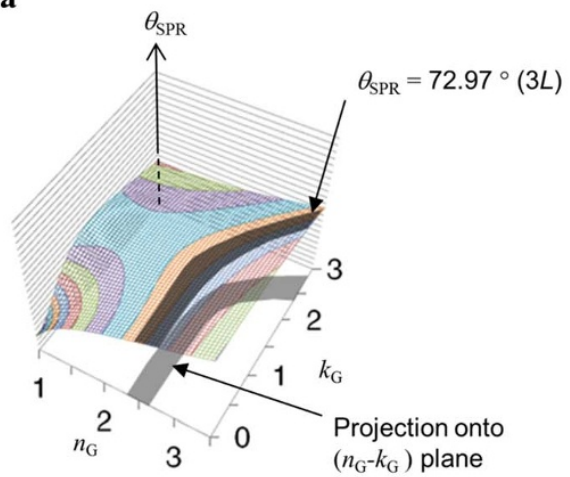

b

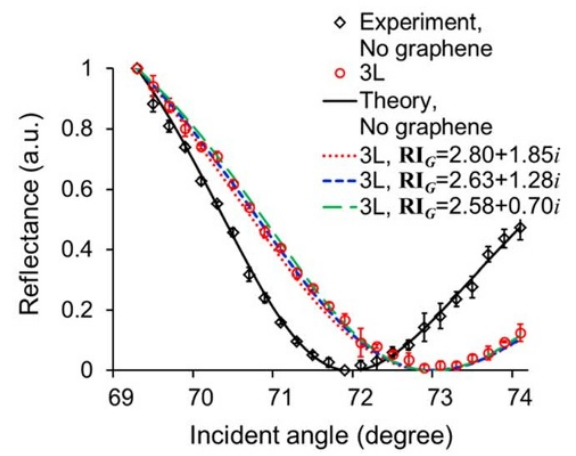

Figure $4 \mid$ Uncertainties associated with the single constraint of $\theta_{\mathrm{SPR}}$ data. (a) The measured SPR angle, i.e., $\theta_{\mathrm{SPR}}=72.97^{\circ}$, is shown as the solid curve (black) on the calculated 3-D contour (SI1) and its projection onto the $\left(n_{G}, k_{G}\right)$ plane (the gray curve) corresponds to all of the fitted solution candidates for $\mathrm{RI}_{G}=n_{G}+i k_{G}$, under the given single constraint, and (b) three arbitrarily selected pairs of $\left(n_{G}, k_{G}\right)$ among these solution candidates predict an identical surface plasmon reflectance curve (SI1), thereby an identical $\theta_{\mathrm{SPR}}$, and all of them fit to the experimental $\theta_{\mathrm{SPR}}$ data, which demonstrates the difficulty in uniquely determining the complex RI of graphene using $\theta_{\mathrm{SPR}}$ data alone.

escent wave is partially attenuated and the resulting reflectance reduces from unity. Furthermore, the amount of reduction in reflectance is different for the $p$-polarized and the $s$-polarized incident light and the ratio of $R_{\mathrm{p}} / R_{\mathrm{s}}$ is dependent upon the incident ray angle (Fig. 1e). The maximum $R_{\mathrm{p}} / R_{\mathrm{s}}$ at the critical angle is selected as a second independent observable to provide an additional constraint for the unique determination of $\left(n_{G}, k_{G}\right)$. As expected, the measured value of maximum $R_{\mathrm{p}} / R_{\mathrm{s}}=1.124$ satisfies multiple pairs of $\left(n_{G}, k_{G}\right)$ as shown in yellow in Fig. 1g, and again, unique determination of complex RI is not possible by this constraint alone. Now, overlapping Fig. If and Fig. 1g creates the intersection region that simultaneously satisfies both constraints of $\theta_{\mathrm{SPR}}$ and maximum $R_{\mathrm{p}} / R_{\mathrm{s}}($ Fig. $1 \mathrm{~h})$, and therefore, the complex graphene $\mathrm{RI}$ is determined to be $\mathrm{RI}_{G}=2.63+$ $1.28 i$, for the case of the three-layered graphene $(L=3)$, which is indeed the average of the two matched pairs corresponding to the intersection.

The resulting $\mathrm{RI}_{G}$ data are shown in Fig. 2 as $n_{G}+i k_{G}=2.58+$ $1.30 i, 2.63+1.28 i$, and $2.73+1.23 i$ for $L=1,3$, and 5 , respectively. The average of these three gives $\mathrm{RI}_{G}=2.65+1.27 \mathrm{i}$ and the variations for different $L$ remain within $\pm 3 \%$ from the average. The smaller uncertainty range for the higher $L$ is attributed mainly to the higher RI sensitivity of $\theta_{\mathrm{SPR}}$ with increasing $L$, and this will be further elaborated in the Discussion section. Calculations are also conducted to predict $\mathrm{RI}_{G}$ using the density function theory (DFT) and the present result of $\mathrm{RI}_{G}=2.71+1.41 \mathrm{i}$ is marked with the star symbol in Fig. 2. In comparison with the published DFT results of $2.96+1.49 i$ for graphene ${ }^{12}$ and $2.88+1.50 i$ for graphite ${ }^{12 \dagger}$, the discrepancies are attributed to the minor differences of the calculation details such as the cutoff energy and the atomic potential modeling, while the dielectric functions agree well for the photon energy up to $20 \mathrm{eV}$.

Also presented in Fig. 2 are published graphene RI data obtained from one of the single measurement techniques imposed with additional conditions and/or assumptions. These data are scattered for the real part, $2.0 \leq n_{G} \leq 3.2$ ( $\pm 23 \%$ scattering), as well as for the imaginary part, $0.78 \leq k_{G} \leq 1.6$ ( $\pm 34 \%$ scattering). The two results from reflection spectroscopy ${ }^{2,3}$ show large deviations in their real parts of $\mathrm{RI}_{G}$ in particular. The former used a constant graphene RI model $^{2}$ that was later proven to be inappropriate ${ }^{4,5,7-9,12}$. The latter ${ }^{3}$ used an incomplete dispersion model under the assumption of the universal optical conductance ${ }^{1}$ and assumed a constant of the real part of the graphene RI as $n_{\mathrm{G}}=3$ for the best fit. Later, this was found to be inaccurate from the more up-to-date DFT analysis as well as from some experimental findings ${ }^{4,5,7-9,12}$. The deviation of the ellipsometry result ${ }^{6}$ from those using a similar ellipsometry technique $e^{4,5,7,8}$ may be attributed to the simplified Drude model used for the data inversion, whereas the latter papers used the Cauchy model ${ }^{4}$, Fano resonance model ${ }^{5}$, point-to-point fitting/Lorentz oscillator $\operatorname{model}^{7}$, and B-spline method ${ }^{8}$, respectively.

Also, the discrepancies of some experimental results may be due to their uses of different samples other than pristine graphene layers, such as reduced graphene oxide (rGO) flakes ${ }^{11 *}$ or highly oriented pyrolytic graphite (HOPG) $)^{15 \dagger}$. Graphene oxide flakes are subjected to form segmented layers with impurities that can lead to reduction of a

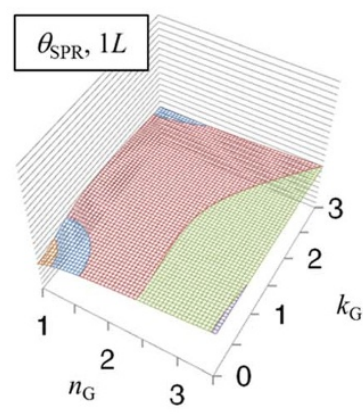

b

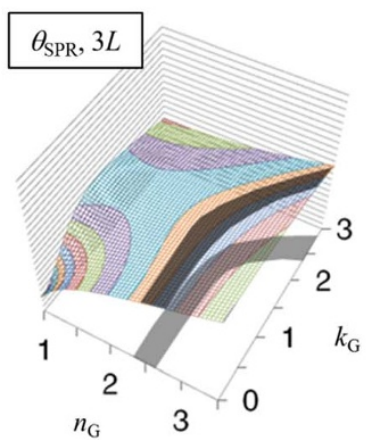

c

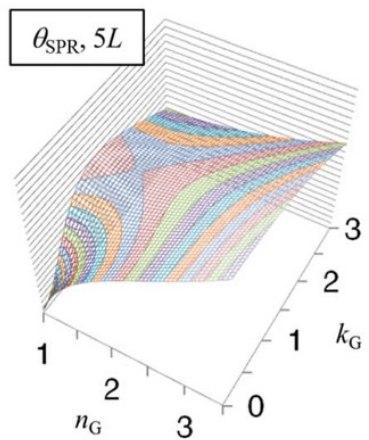

Figure $5 \mid$ Measurement sensitivity of $\boldsymbol{\theta}_{\mathrm{SPR}}$ vs. the uncertainties of $\left(\boldsymbol{n}_{G}, \boldsymbol{k}_{G}\right)$. The predicted $\theta_{\mathrm{SPR}}$ contours (SI1) with consistent $0.2^{\circ}$ increments are shown for $L=1,3$, and 5 , and the slope of the contours above the $\left(n_{G}, k_{G}\right)$ plane increases with increasing $L$. This implies that, given a fixed measurement sensitivity of $\pm 0.1^{\circ}$ for $\theta_{\mathrm{SPR}}$, the corresponding uncertainties in determining $\left(n_{G}, k_{G}\right)$ are reduced with increasing $L$. 
$\mathbf{a}$

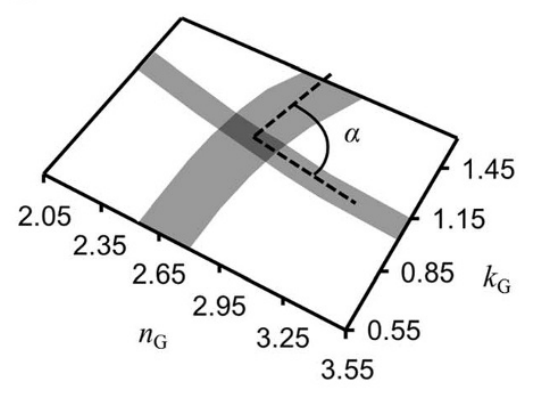

b

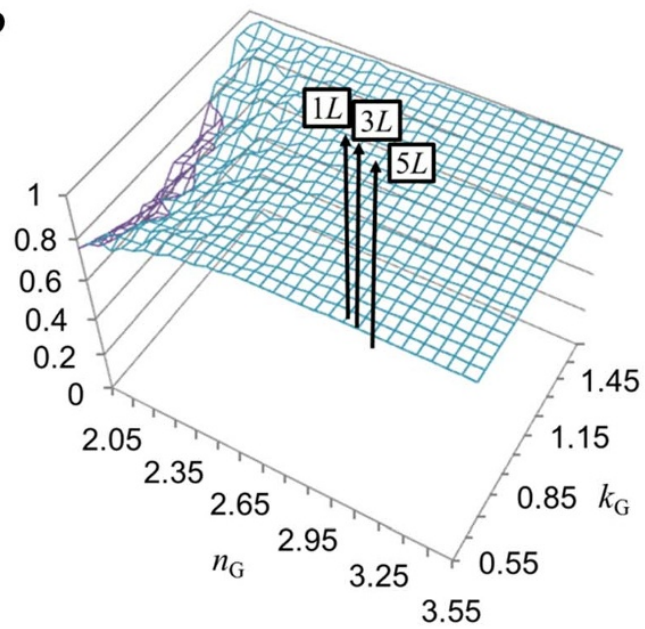

Figure 6 Orthogonality of the two independent observables, $\boldsymbol{\theta}_{\mathrm{SPR}}$ and $\boldsymbol{R}_{\mathrm{p}} / \boldsymbol{R}_{\mathrm{s}}{ }^{*}$. (a) The orthogonality of the two sets of solution pairs $\left(n_{\mathrm{G}}, k_{\mathrm{G}}\right)$ from the two constraints, $\theta_{\mathrm{SPR}}$ and $R_{\mathrm{p}} / R_{\mathrm{s}}{ }^{*}$, is defined as the sine function of their intersection angle $\alpha$ on the projected plane, and (b) the maximum orthogonality of unity for $\alpha=90^{\circ}$ provides the strong independence of the two constraints ensuring the unique determination of the complex RI of graphene. All of the three measured RI values for $L=1,3$, and 5 fall on the desirable maximum orthogonality of unity.

electron mobility, and equivalently, reduced imaginary part of RI $\left(k_{G}\right)$. In contrast, the agility of electrons in the highest-grade HOPG can result in high optical conductivity and a noticeably large $k_{G}$.

\section{Discussion}

Figure 3a illustrates the fitting uncertainties of the complex graphene RI to the measured $R_{p} / R_{s}$ for the case of the $1 L$ graphene sample. The three curves correspond to three arbitrarily selected pairs of $\left(n_{G}, k_{G}\right)$ from the solution candidate pool (Fig. 1g) and all three curves fit to the measured maximum $R_{p} / R_{s}{ }^{*}=1.124$. This implies that any of the three pairs, $(3.45+1.00 i),(2.63+1.28 i)$ or $(1.90+1.90 i)$ can be accepted as a fitted value for the complex graphene RI. Indeed, projection of the measured constraint $R_{p} / R_{s}^{*}=1.124$ onto the $n_{G}-k_{G}$ plane (Fig. 3b) shows infinite pairs of $\left(n_{G}, k_{G}\right)$ that satisfy the measured constraint. Measurements of SPR reflectance (Fig. 4a) also show that all three curves generated with three arbitrarily selected $(2.80+1.85 i),(2.63+1.28 i)$ or $(2.58+0.70 i)$ fit closely to the measured and $\theta_{\mathrm{SPR}}=72.97^{\circ}$. Again, the $n_{G}-k_{G}$ projection of the corresponding contour of measured $\theta_{\mathrm{SPR}}=72.97^{\circ}$ provides only a partial constraint that any of these pairs of $\left(n_{G}, k_{G}\right)$ located on the projection band can be accepted for the fitting solution.

The contacts between graphene and a variety of metals were discussed by theoretically investigating the Fermi level shift in the contacted graphene from the freestanding one, and calculating the electronic structure and electrostatic potential ${ }^{16}$. Their findings include that for the Au-graphene contact, there lies a potential barrier between them, which will cause extra contact resistance, resulting in only weak physical contact. Thus, we believe that graphene maintains weak physical contact with the gold substrate and that the opto-electric properties of graphene, including its complex RI, are not altered by the contacting $\mathrm{Au}$ substrate. Furthermore, we have found that a number of research groups have used different substrates contacting graphene, assuming no significant changes in graphene's RI properties for the case of amorphous quartz substrate, GaAs substrate ${ }^{6}$, poly-dimethyl-siloxane (PDMS) ${ }^{10}$, and also for $\mathrm{Au}$ substrate ${ }^{11}$.

For AA-stacking of multilayer graphene, i.e., atom-to-atom arrangement of graphene layers, no measurable distinctions in RI were observed with the number of graphene layers ${ }^{17}$. For twisted bilayer graphene including $\mathrm{AB}$-stacking ${ }^{18,19}$, in contrast, the reflection contrast of multilayers was found to slightly vary with the existence of the $2^{\text {nd }}$ graphene layer; however, more consistent and quantitative conclusions are still under examination. Note that these findings were relevant for mechanically exfoliated graphene layers from highly ordered pyrolytic graphite (HOPG), whereas the present study examined CVD-synthesized graphene layers that were created by the crystallized growth of many randomly oriented
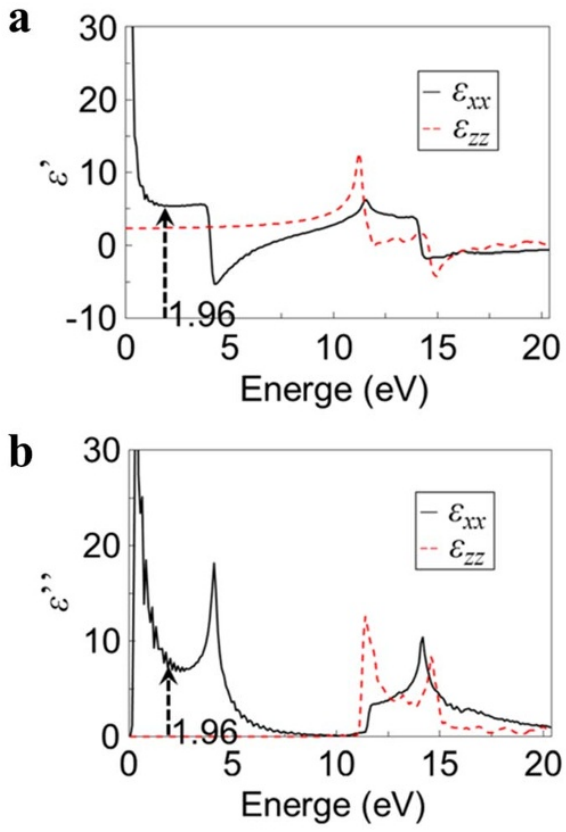

Figure $7 \mid$ Ab-initio DFT calculation results for the complex dielectric function of graphene. The real (a) and imaginary (b) parts of the complex dielectric function of pristine graphene are calculated using the density functional theory (DFT). The dielectric function $\varepsilon_{x x}$ is for the electric field oscillating parallel to the graphene layer and $\varepsilon_{z z}$ is for the electric field perpendicular to the graphene layer. Note that our proposed scheme, like all other available experimental methods, is able to conduct measurements only for the $x x$-components of the complex RI where the electric field oscillates in parallel with the graphene layer and is unable to detect the $z z$ components. For the incident wave of $634 \mathrm{~nm}$ corresponding to $1.96 \mathrm{eV}$, the real and imaginary parts of the relevant dielectric function are given by $\varepsilon_{x x}=5.36+7.64 i$ and the resulting complex RI of graphene is given by $\sqrt{\varepsilon_{x x}}=n_{G}+i k_{G}=2.71+1.41 i$. 
submicron-sized grains. Therefore, each layer of CVD-synthesized graphene is considered to be randomly oriented, and the multilayered graphene obtained by repeated transfer of each layer does not provide any consistent stacking orientations.

The increased total thickness of graphene with increasing $L$ creates steeper contour surfaces above the $n_{G}-k_{G}$ plane (Fig. 5), which in turn contributes to lowering the measurement uncertainties. The smaller error bars of the measured complex RI data with increasing $L$, as shown in Fig. 2, are attributed to this enhanced sensitivity with increased total thickness of the graphene samples. It is worthwhile noting that the multi-layered graphene samples are prepared by physical stacking of multiple CVD-synthesized layers, one at a time, through multi-step processing such as spin coating, baking, and etching of PMMA and use of chemical ${ }^{20}$ and extra care must be taken to minimize left-over impurities and contaminants. It is worth noting that a small amount of impurities would make discernible changes in the measured $\theta_{\mathrm{SPR}}$ resulting in an overestimated $n_{\mathrm{G}}$ since the SPR is known as the most sensitive detection tool for the real component of $\mathrm{R}^{21}$. In addition, the Raman signal can provide quite reliable information on imperfections, disorders, and grain boundaries on graphene. However, the PMMA residues that may result from incomplete washing after the transfer process are not readily detectable by Raman signal since they are Raman inactive ${ }^{22}$.

Independence of the two observables, $\theta_{\mathrm{SPR}}$ for minimum SPR reflectance and the maximum $R_{p} / R_{s}$ for attenuated reflection, can be assessed by their mutual orthogonality on the $n_{G}-k_{G}$ plane (Fig. 6), which is defined as a sine function of the intersection angle of the two projected contour bands. Thus, the orthogonalities of near unity of the present cases of $L=1,3$, or 5 supports fairly unique determinations of the graphene RI within acceptable experimental uncertainties. Also, the fitting uncertainties for both SPR angles and total reflectance ratios associated with FWHM of $22 \mathrm{~nm}$ are shown to be less than $0.3 \%$ deviations from those with zero FWHM, based on the reflection calculations presented in Supplementary Information.

\section{Methods}

Preparation of graphene sample on a partially Au-coated glass substrate. Single graphene layers were grown on thin Cu foils ( $25 \mu \mathrm{m}$ thick, $99.9999 \%$ purity by Alfa Aesar Inc.) using a commercially available low-pressure-high-temperature (LPHT) chemical vapor deposition (CVD) system by ScienTech Inc. A portion of the slide glass substrate was masked with a cover slip using Kapton tape (DuPont and Kapton Inc.) before the adhesion layer of $\mathrm{Cr}(4 \mathrm{~nm})$ and Au thin layer $(48 \mathrm{~nm})$ were sequentially sputtered. Then, the cover slip was removed to recover the region of uncoated glass substrate. Finally, CVD graphene layers were transferred onto the substrate using a conventional PMMA (poly-methyl methacrylate) process ${ }^{20}$ and the final test sample was completed as shown in Fig. 1b.

Incident angle measurements. The angle of incidence was measured by a Pro 3600, Mitutoyo digital protractor with $0.01^{\circ}$ minimum reading scale. The tilt angles of the two arms of the experimental system (Fig. 1a) were measured with respect to the horizon and the actual incident angle to the graphene sample placed on top of the BK7 prism was accounted for the refraction of the incident ray into the prism. The measurement increments ranged from 0.1 to $0.7^{\circ}$ to accommodate minimum distinguishable measurements of the reflectance data.

Data acquisition and recording. The reflected light intensity was detected by a Princeton Instrument ProEM 512b EM-CCD (electron-multiplied charge-coupled device) with $512 \times 51216$-micron-square pixels at a 16-bit data transfer rate. The non-linearity of the CCD due to the low-noise was kept under $1 \%$ and the coolant temperature was kept at $-70^{\circ} \mathrm{C}$.

Calculations of complex RI of graphene using Density Function Theory (DFT). Our calculations for complex graphene RI used the Vienna Ab-initio Simulation Package (VASP) to determine the dielectric constants for both the in-plane $(x x)$ and cross-plane ( $z z$ ) directions (Fig. 7). Then, the complex RI value of graphene can be readily obtained by taking a square root of a complex dielectric constant at a specific energy (equivalently, wavelength ${ }^{-1}$ ). The charge density distributions were first calculated for use in the electronic band structure and dielectric function calculations. In calculating the charge distributions, graphene was set to have a constant interatomic distance of $1.42 \AA$ and the tetrahedron method with Blöchl corrections was used for partial occupancy with broadening of $0.1 \mathrm{eV}$. The projector-augmented wave (PAW) method was adopted with ultra-soft pseudo-potentials and energy cutoff of
$400 \mathrm{eV}$. The K-point grid was generated by automatic generation of $\Gamma$-centered $36 \times$ $36 \times 12$ mesh and the electronic band structures were calculated with Gaussian smearing along the high symmetry points of the irreducible Brillouin zone of graphene, $\Gamma-\mathrm{M}-\mathrm{K}-\Gamma$, with 20 segments in each line. The present result shown by the star symbol in Fig. 2 comes from consideration of the dielectric constant at $1.96 \mathrm{eV}$, which corresponds to the center wavelength of $634 \mathrm{~nm}$ of the LED light that was used for the experiment.

1. Nair, R. R. et al. Fine structure constant defines visual transparency of graphene. Science 320, 1308 (2008).

2. Ni, Z. H. et al. Graphene thickness determination using reflection and contrast spectroscopy. Nano Lett. 7, 2758-2763 (2007).

3. Bruna, M. \& Borini, S. Optical constants of graphene layers in the visible range. Appl. Phys. Lett. 94, 031901-031903 (2009).

4. Kravets, V. G. et al. Spectroscopic ellipsometry of graphene and an exciton-shifted van Hove peak in absorption. Phys. Rev. B. 81, 021912 (2010).

5. Matkovic, A. et al. Spectroscopic imaging ellipsometry and Fano resonance modeling of graphene. J. Appl. Phys. 112, 123523 (2012).

6. Wurstbauer, U. et al. Imaging ellipsometry of graphene. Appl. Phys. Lett. 97, 231901 (2010).

7. Nelson, F. J. et al. Optical properties of large-area polycrystalline chemical vapor deposited graphene by spectroscopic ellipsometry. Appl. Phys. Lett. 97, 253110 (2010).

8. Weber, J. W., Calado, V. E. \& van de Sanden, M. C. M. Optical constants of graphene measured by spectroscopic ellipsometry. Appl. Phys. Lett. 97, 091904 (2010).

9. Wang, X., Chen, Y. P. \& Nolte, D. D. Strong anomalous optical dispersion of graphene: complex refractive index measured by Picometrology. Opt. Express 16, 22105-22112 (2008).

10. Ye, Q. et al. Polarization-dependent optical absorption of graphene under total internal reflection. Appl. Phys. Lett. 102, 021912 (2013).

11. Xue, T. et al. A switch of the oxidation state of graphene oxide on a surface plasmon resonance chip. ACS Appl. Mater. Interfaces 5, 2096-2103 (2013).

12. Klintenberg, M. et al. Evolving properties of two-dimensional materials: from graphene to graphite. J. Phys.: Condens. Matter 21, 335502 (2009).

13. Kihm, K. D. Ch. 5. Surface Plasmon Resonance Microscopy (SPRM). Near-Field Characterization of Micro/Nano-Scaled Fluid Flows. (Springer-Verlag, Berlin Heidelberg, 2011)

14. Kihm, K. D. et al. Surface plasmon resonance (SPR) reflectance imaging: Far-field recognition of near-field phenomena. Opt. Laser. Eng. 50, 64-73 (2012).

15. Jellison, Jr, G. E., Hunn, J. D. \& Lee, H. N. Measurement of optical functions of highy oriented pyrolytic graphite in the visible. Phys. Rev. B 76, 085125 (2007).

16. Ran, Q., Gao, M., Guan, X., Wang, Y. \& Yu, Z. First-principles investigation on bonding formation and electronic structure of metal-graphene contacts. Appl. Phys. Lett. 94, 103511 (2009).

17. Chiu, C. W., Chen, S. C., Huang, Y. C., Shyu, F. L. \& Lin, M. F. Critical optical properties of AA-stacked multilayer graphenes. Appl. Phys. Lett. 103, 041907 (2013).

18. Wang, Y. et al. Stacking dependent optical conductivity of bilayer graphene. ACS Nano 4, 4074-4080 (2010).

19. Campos-Delgado, J., Algara-Siller, G., Santos, C. N., Kaiser, U. \& Raskin, J.-P. Twisted bi-layer graphene: microscopic rainbows. Small 9, 3247-3251 (2013).

20. Li, X. et al. Large-Area Synthesis of High-Quality and Uniform Graphene Films on Copper Foils. Science 324, 1312-1314 (2009).

21. White, Ian, M. \& Fan, X. On the performance quantification of resonant refractive index sensors. Opt. Express 16, 1020-1028 (2008).

22. Xu, W. et al. Surface enhanced Raman spectroscopy on a flat graphene surface. P. Natl. Acad. Sci. USA 109, 9281-9286 (2012).

\section{Acknowledgments}

This research work was supported partially by the World Class University (WCU) Program (R31-2008-000-10083-0) and partially by the Nano-Material Technology Development Program (R2011-003-2009), both through the National Research Foundation of Korea (NRF) funded by the Ministry of Science, ICT and Future Planning.

\section{Author contributions}

S.C. and K.D.K. designed, carried out the experiments, acquired, analyzed the data, executed the calculations, and wrote the manuscript; H.G.K. synthesized the graphene layers using the CVD method; G.L. prepared for the graphene layer samples transferred from the $\mathrm{Cu}$ foils onto the partially Au-coated glass substrates; J.S.P. contributed to the design and alignment of the optical layout of the experimental system; J.S.L. helped with the idea of fundamental analysis and overall supervision of the project.

\section{Additional information}

Supplementary information accompanies this paper at http://www.nature.com/ scientificreports 
Competing financial interests: The authors declare no competing financial interests.

How to cite this article: Cheon, S. et al. How to Reliably Determine the Complex Refractive Index (RI) of Graphene by Using Two Independent Measurement Constraints. Sci. Rep. 4, 6364; DOI:10.1038/srep06364 (2014).
(F) $\Theta$ This work is licensed under a Creative Commons Attribution-NonCommercialNoDerivs 4.0 International License. The images or other third party material in this article are included in the article's Creative Commons license, unless indicated otherwise in the credit line; if the material is not included under the Creative Commons license, users will need to obtain permission from the license holder in order to reproduce the material. To view a copy of this license, visit http:// creativecommons.org/licenses/by-nc-nd/4.0/ 